\title{
Angioscopic Assessments at 3 Months After Fluoropolymer-Based Paclitaxel-Eluting Stent Implantation for Femoropopliteal Endovascular Intervention
}

\author{
Takuya Tsujimura, MD; Takayuki Ishihara, MD; Osamu Iida, MD; \\ Yosuke Hata, MD; Naoya Kurata; Mitsutoshi Asai, MD, PhD; \\ Masaharu Masuda, MD, PhD; Shin Okamoto, MD; Kiyonori Nanto, MD; \\ Takashi Kanda, MD; Yasuhiro Matsuda, MD; Toshiaki Mano, MD, PhD
}

Background: The detailed mechanism of early-phase arterial healing after novel fluoropolymer-based paclitaxel-eluting stent (PES) implantation in the femoropopliteal (FP) lesions has not been elucidated.

Methods and Results: We evaluated the intravascular status of 20 PES implanted in 11 FP lesions of 9 patients using angioscopy at approximately 3 months after implantation. Angioscopic images were analyzed to determine (1) the dominant degree of neointimal coverage (NIC) over the stent; (2) the extent of uncovered struts; and (3) the presence of intrastent thrombus. NIC was classified into 4 grades: grade 0 , stent struts fully visible; grade 1, stent struts bulging into the lumen although covered; grade 2, stent struts embedded in neointima, but translucently visible; grade 3, stent struts fully embedded and invisible. The extent of uncovered struts was scored as follows: score 0 , no uncovered struts of the entire stent; score 1 , uncovered struts area approximately $<30 \%$ of the stent; and score 2, uncovered struts area approximately $\geq 30 \%$ of the stent. In total, $90 \%$ of stents demonstrated grade 1 dominant NIC and $10 \%$ showed grade 2 dominant NIC; $85 \%$ of stents showed an uncovered stent score of 0 , and the remainder had a score of 1 . Thrombus was observed in all stents.

Conclusions: Widely uncovered stent struts were not observed by angioscopy at 3 months after PES implantation in these FP lesions, even with the detection of thrombus adhesion.

Key Words: Angioscopy; Drug-eluting stents; Endovascular therapy; Peripheral artery disease

$\mathbf{E}$ ndovascular therapy (EVT) is widely used in the management of symptomatic peripheral artery disease (PAD). As a result of technical advancements and device development, more complex lesions can be indicated for EVT. Unfortunately, luminal narrowing (i.e., restenosis) continues to limit the long-term success of the traditional procedure, especially in femoropopliteal (FP) lesions, requiring repeat revascularization within 12 months in approximately $20-40 \%$ of patients. ${ }^{1,2}$ Indeed, restenosis might be considered the Achilles heel of FP-EVT.

The IMPERIAL randomized trial demonstrated that a novel fluoropolymer-based paclitaxel-eluting stent (PES) (Eluvia; Boston Scientific, Marlborough, MA, USA) was superior to a polymer-free paclitaxel-coated stent (PCS) (Zilver PTX; Cook Corporation, Bloomington, IN, USA) in terms of 12-month primary patency and major adverse events. ${ }^{3}$ However, the detailed mechanism of this difference has not been elucidated. In addition, it is clinically important to evaluate intravascular status using intravascular imaging devices after the intervention. Of these, angioscopy is the only method that can evaluate intravascular status in vivo with real images and in full color. ${ }^{4-9}$ Therefore, we sought to investigate the intravascular status using angioscopy to evaluate early-phase arterial healing after PES implantation.

\section{Patients}

This was a single-center, prospective observational study. From May 2019 to November 2019, we attempted to angioscopically evaluate the intravascular status of 22 PES implanted in 11 lesions of 9 patients approximately 3 months after implantation. All PES with angioscopic

Received May 29, 2020; revised manuscript received July 7, 2020; accepted July 15, 2020; J-STAGE Advance Publication released online September 26, 2020 Time for primary review: 17 days

Kansai Rosai Hospital Cardiovascular Center, Amagasaki (T.T., T.I., O.I., Y.H., M.A., M.M., S.O., K.N., T.K., Y.M., T.M.); Department of Clinical Engineering, Kansai Rosai Hospital, Amagasaki (N.K.), Japan

Mailing address: Takayuki Ishihara, MD, Kansai Rosai Hospital Cardiovascular Center, 3-1-69 Inabaso, Amagasaki 660-8511, Japan. E-mail: t.ishihara31@gmail.com

All rights are reserved to the Japanese Circulation Society. For permissions, please e-mail: cj@j-circ.or.jp

ISSN-1346-9843 


\section{Definition of Neointimal Coverage Grade}

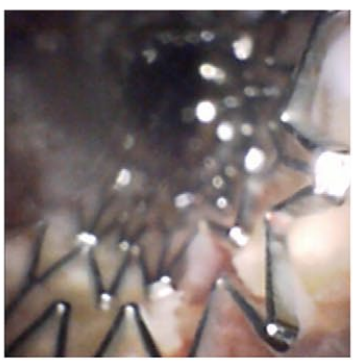

Grade 0

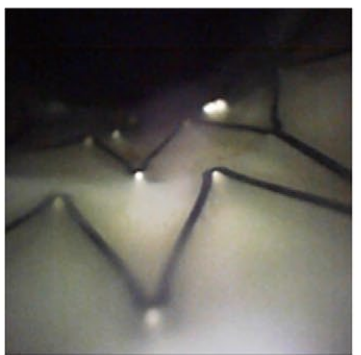

Grade 1

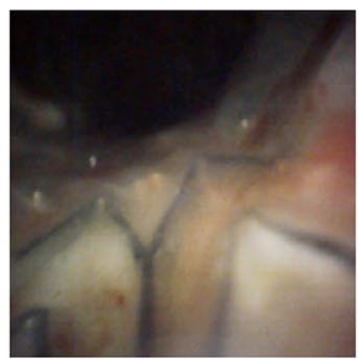

Grade 2

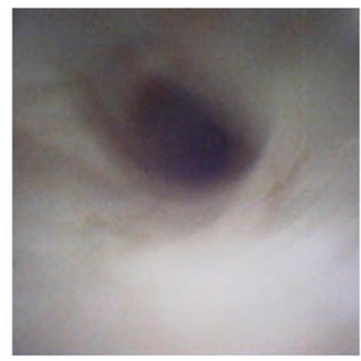

Grade 3

Grade 0: stent struts exposed

Grade 1: struts bulged into the lumen, although covered

Grade 2: struts embedded by the neointima, but translucent

Grade 3: struts fully embedded and invisible

Figure 1. Classification of neointimal coverage into 4 grades: grade 0 , stent struts fully visible; grade 1 , stent struts bulging into the lumen although covered; grade 2, stent struts embedded in the neointima, but translucently visible; grade 3, stent struts fully embedded and invisible.

\section{Definition of Uncover Score}

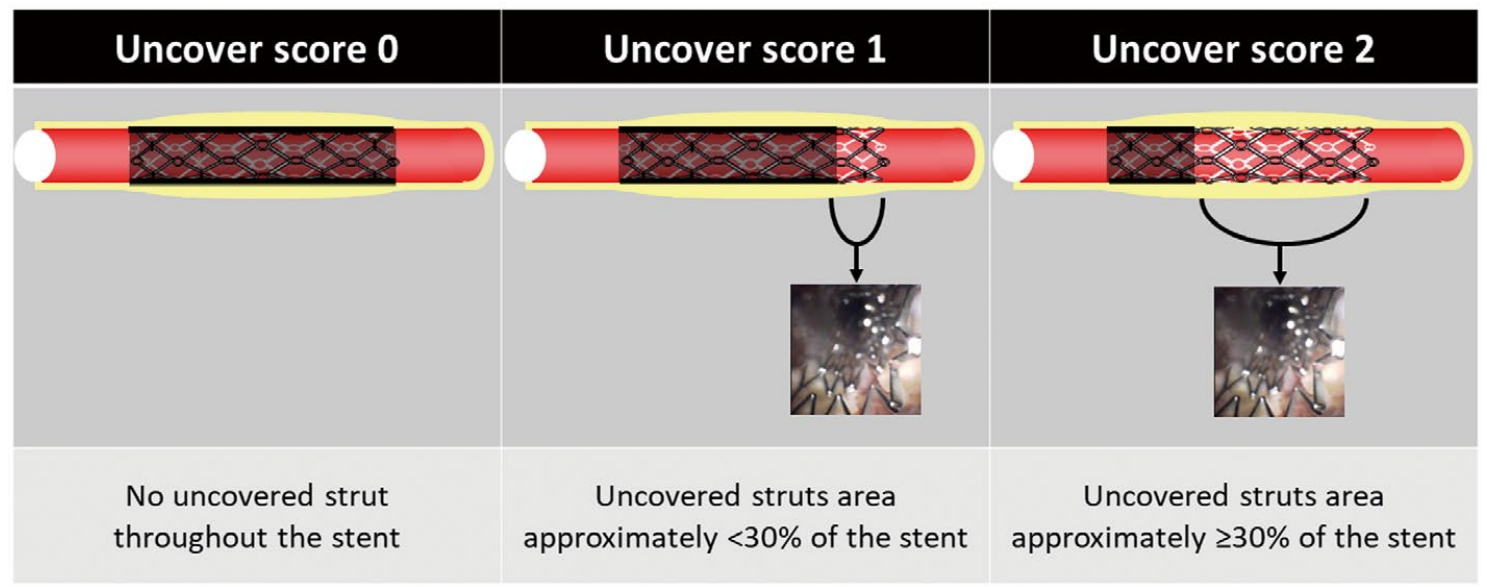

Figure 2. The extent of uncovered struts was scored as follows: uncover score 0 , no uncovered struts throughout the stent; uncover score 1 , uncovered struts area approximately $<30 \%$ of the stent; uncover score 2 , uncovered struts area approximately $\geq 30 \%$ of the stent.

evaluation were implanted in de novo lesions in native FP arteries. Although angioscopic evaluation in the early phase was recommended for all patients with staged or required EVT for other lesions, it was not performed when informed consent could not be obtained, or when a specialist in angioscopic evaluation was not available. In principle, the elective patients received clopidogrel $(75 \mathrm{mg} /$ day $)$ or prasugrel $(3.75 \mathrm{mg} /$ day $)$ in addition to aspirin $(100 \mathrm{mg} /$ day $)$ at least 1 week before EVT, and continued to receive dual antiplatelet therapy during the follow-up period at the discretion of their attending physicians. In order to decrease the bleeding risk in some cases, dual antiplatelet therapy was switched to single antiplatelet therapy after EVT if the patient was taking an anticoagulant drug. This study was performed in accordance with the Declaration of Helsinki. The Medical Ethics Committee of Kansai Rosai Hospital 


\begin{tabular}{|c|c|c|c|c|c|c|c|c|c|}
\hline & \multicolumn{9}{|c|}{ Patient no. } \\
\hline & 1 & 2 & 3 & 4 & 5 & 6 & 7 & 8 & 9 \\
\hline Age, years & 76 & 84 & 62 & 62 & 75 & 79 & 81 & 76 & 97 \\
\hline Sex & Male & Female & Female & Male & Female & Male & Female & Male & Female \\
\hline $\mathrm{BMI}, \mathrm{kg} / \mathrm{m}^{2}$ & 23.5 & 26.7 & 24.3 & 22.2 & 26.4 & 24.0 & 24.2 & 20.5 & 20.3 \\
\hline Rutherford class & 6 & 5 & 2 & 5 & 5 & 5 & 5 & 6 & 5 \\
\hline Hypertension & + & + & + & + & - & + & + & + & - \\
\hline Dyslipidemia & + & + & + & + & - & + & - & + & + \\
\hline Diabetes mellitus & + & + & + & - & + & + & + & - & - \\
\hline CKD & + & + & + & + & + & + & + & - & + \\
\hline Hemodialysis & - & + & + & + & + & + & + & - & - \\
\hline Current smoking & - & - & - & - & - & - & - & - & - \\
\hline CAD & + & - & + & - & + & + & + & - & - \\
\hline Stroke & - & - & - & - & & + & - & - & - \\
\hline Aspirin use & + & + & + & + & + & + & + & + & + \\
\hline Clopidogrel use & + & + & - & + & - & + & - & + & + \\
\hline Prasugrel use & - & - & + & - & - & - & - & - & - \\
\hline Cilostazol use & - & - & - & - & - & - & - & - & - \\
\hline Warfarin use & - & - & - & - & + & - & + & - & - \\
\hline DOAC use & - & - & - & - & - & - & - & - & - \\
\hline Statin use & + & - & + & - & - & - & - & - & + \\
\hline
\end{tabular}

+, present; -, absent. BMI, body mass index; CAD, coronary artery disease; CKD, chronic kidney disease; DOAC, direct oral anticoagulant.

\begin{tabular}{|c|c|c|c|c|c|c|c|c|c|c|c|}
\hline & \multicolumn{11}{|c|}{ Lesion number } \\
\hline & 1 & 2 & 3-1 & $3-2$ & 4-1 & $4-2$ & 5 & 6 & 7 & 8 & 9 \\
\hline Lesion side & Right & Left & Right & Right & Right & Left & Right & Left & Left & Left & Left \\
\hline TASC II classification & D & D & D & $\mathrm{C}$ & $\mathrm{D}$ & B & $\mathrm{D}$ & $\mathrm{D}$ & $A$ & B & $\mathrm{D}$ \\
\hline Inclusion of popliteal artery lesion & + & - & - & + & + & - & - & - & - & - & - \\
\hline Chronic total occlusion & - & - & + & - & - & - & - & - & - & - & + \\
\hline PACSS grade & 1 & 3 & 4 & 4 & 3 & 1 & 4 & 3 & 3 & 3 & 1 \\
\hline Lesion length, $\mathrm{cm}$ & 35 & 22 & 35 & 20 & 5 & 17 & 24 & 10 & 4 & 23 & 23 \\
\hline Distal reference vessel diameter, $\mathrm{mm}$ & 6.3 & 4.4 & 4.3 & 4.0 & 5.0 & 5.6 & 5.3 & 5.8 & 4.0 & 3.5 & 5.2 \\
\hline No. of BK run off & 0 & 2 & 1 & 1 & 1 & 2 & 1 & 1 & 1 & 1 & 1 \\
\hline
\end{tabular}

+, present; -, absent. BK, below-the-knee; PACCS, peripheral artery calcification scoring system; TASC, Trans-Atlantic Inter-Society Consensus.

approved the study, and all patients provided written informed consent.

\section{Angiographic and Angioscopic Follow-up}

After the administration of unfractionated heparin $(5,000$ IU) into the femoral artery via the inserted sheath, angioscopy was performed using a Zemporshe ${ }^{\circledR}$ angioscope (OVALIS, Osaka, Japan). Briefly, an optical fiber was placed in the distal segment of the FP artery slightly protruding from the guiding sheath, and then manually pulled back from the distal edge of the stent to the proximal edge under careful angioscopic and angiographic guidance while blocking blood flow by flushing with low-molecularweight dextran. Angioscopic images were made up of 480,000 pixels in full color and were digitally stored for offline analysis.

\section{Angioscopic Analysis and Outcome Measures}

Angioscopic images were analyzed for each stent to determine (1) the dominant degree of neointimal coverage (NIC) over the stent; (2) the heterogeneity of NIC; (3) the extent of uncovered struts; and (4) the presence of intrastent thrombus. NIC over the stent was classified into 4 grades as previously described: grade 0 , stent struts fully visible, similar to the status immediately after implantation; grade 1 , stent struts bulging into the lumen, although covered, still translucently visible; grade 2 , stent struts embedded in the neointima, but translucently visible; grade 3 , stent struts fully embedded and invisible on angioscopy ${ }^{4}$ (Figure 1). Heterogeneity of NIC has been defined previously as follows: ${ }^{4}$ homogeneity, NIC grade is uniform in the entire stented segment; heterogeneity of 1 grade, different NIC grades of 1 grade were present in the entire stented segment; heterogeneity of 2 grades, different NIC grades of 2 grades were present in the entire stented segment; different NIC grades of 3 grades were present in the entire stented segment. NIC was judged as heterogeneous when differences in the NIC grade became apparent. Struts located in the overlapped segment were excluded from grading, and stent edges were excluded from the heterogeneity analysis. The 


\begin{tabular}{|c|c|c|c|c|c|c|c|c|c|c|c|}
\hline & \multicolumn{11}{|c|}{ Stent number } \\
\hline & $1-1$ & $1-2$ & $1-3$ & $2-1$ & $2-2$ & 3-1-1 & $3-1-2$ & $3-2-1$ & $3-2-2$ & 4-1-1 & $4-1-2$ \\
\hline Follow-up period, days & 67 & 67 & 67 & 101 & 101 & 104 & 104 & 98 & 98 & 117 & 117 \\
\hline Diameter of predilatation balloon, $\mathrm{mm}$ & 7.0 & 7.0 & 7.0 & 6.0 & 6.0 & 5.0 & 5.0 & 5.5 & 5.5 & 6.0 & 6.0 \\
\hline Stent diameter, $\mathrm{mm}$ & 7.0 & 7.0 & 7.0 & 6.0 & 6.0 & 7.0 & 7.0 & 7.0 & 6.0 & 6.0 & 7.0 \\
\hline Stent length, $\mathrm{cm}$ & 12 & 12 & 12 & 12 & 10 & 12 & 4 & 12 & 12 & 8 & 8 \\
\hline Diameter of postdilatation balloon, $\mathrm{mm}$ & 7.0 & 7.0 & 7.0 & 6.0 & 6.0 & 5.0 & 5.0 & 5.5 & 5.5 & 6.0 & 6.0 \\
\hline
\end{tabular}

\begin{tabular}{lcccccccccccc} 
& \multicolumn{10}{c}{ Stent number } \\
\cline { 2 - 11 } & $\mathbf{4 - 2 - 1}$ & $\mathbf{4 - 2 - 2}$ & $\mathbf{5 - 1}$ & $\mathbf{5 - 2}$ & $\mathbf{5 - 3}$ & $\mathbf{6}$ & $\mathbf{7}$ & $\mathbf{8 - 1}$ & $\mathbf{8 - 2}$ & $\mathbf{9 - 1}$ & $\mathbf{9 - 2}$ \\
Follow-up period, days & 120 & 120 & 110 & 110 & 110 & 75 & 104 & 67 & 67 & 99 & 99 \\
Diameter of predilatation balloon, $\mathrm{mm}$ & 7.0 & 7.0 & 6.0 & 6.0 & 6.0 & 7.0 & 6.0 & 5.0 & 5.0 & 4.0 & 4.0 \\
Stent diameter, mm & 7.0 & 7.0 & 7.0 & 7.0 & 7.0 & 7.0 & 6.0 & 6.0 & 6.0 & 7.0 & 7.0 \\
Stent length, cm & 12 & 8 & 12 & 12 & 8 & 12 & 4 & 12 & 12 & 12 & 12 \\
Diameter of postdilatation balloon, $\mathrm{mm}$ & 7.0 & 7.0 & 6.0 & 6.0 & 6.0 & 7.0 & 6.0 & 6.0 & 6.0 & 6.0 & 6.0 \\
\hline
\end{tabular}

\begin{tabular}{|c|c|c|c|c|c|c|c|c|c|c|c|}
\hline & \multicolumn{11}{|c|}{ Stent number } \\
\hline & $1-1$ & $1-2$ & $1-3$ & $2-1$ & $2-2$ & 3-1-1 & $3-1-2$ & $3-2-1$ & $3-2-2$ & $4-1-1$ & $4-1-2$ \\
\hline Dominant NIC grade & $\mathrm{N} / \mathrm{A}$ & $\mathrm{N} / \mathrm{A}$ & 1 & 1 & 1 & 1 & 1 & 1 & 1 & 1 & 1 \\
\hline Heterogeneity of NIC grade & $\mathrm{N} / \mathrm{A}$ & $\mathrm{N} / \mathrm{A}$ & 1 & 1 & 1 & 0 & 0 & 0 & 0 & 1 & 2 \\
\hline Thrombus adhesion & $\mathrm{N} / \mathrm{A}$ & $\mathrm{N} / \mathrm{A}$ & + & + & + & + & + & + & + & + & + \\
\hline \multirow[t]{2}{*}{ Uncover score } & $\mathrm{N} / \mathrm{A}$ & $\mathrm{N} / \mathrm{A}$ & 0 & 1 & 0 & 0 & 0 & 0 & 0 & 0 & 0 \\
\hline & $4-2-1$ & $4-2-2$ & $5-1$ & $5-2$ & $5-3$ & 6 & 7 & $8-1$ & $8-2$ & $9-1$ & $9-2$ \\
\hline Dominant NIC grade & 1 & 1 & 2 & 1 & 1 & 1 & 2 & 1 & 1 & 1 & 1 \\
\hline Heterogeneity of NIC grade & 1 & 1 & 2 & 1 & 0 & 1 & 1 & 1 & 2 & 1 & 1 \\
\hline Thrombus adhesion & + & + & + & + & + & + & + & + & + & + & + \\
\hline Exposed grade & 0 & 0 & 0 & 0 & 0 & 0 & 0 & 0 & 0 & 1 & 1 \\
\hline
\end{tabular}

+ , present. N/A, not available; NIC, neointimal coverage.

extent of the uncovered struts was scored with reference to the previous definition: uncover score 0 , no uncovered struts throughout the stent; uncover score 1, uncovered struts area approximately $<30 \%$ of the stent; uncover score 2 , uncovered struts area approximately $\geq 30 \%$ of the stent $^{5,10}$ (Figure 2). Thrombus was defined based on criteria adopted by the European Working Group on Coronary Angioscopy. ${ }^{6}$ As described elsewhere, the estimated interand intra-observer $\kappa$ coefficients were 0.84 and 0.95 , respectively, for the dominant degree of NIC over the stent, 0.84 and 0.83 for heterogeneity of NIC and 0.93 and 1.0 for the presence of intrastent thrombus. ${ }^{7}$

\section{Statistical Analysis}

No previous data on angioscopic evaluation of drug-eluting stents in the FP arteries were available to inform our choice of sample size. However, we set 20 PES as the target sample, because this number seemed reasonable for an initial investigation, judging from previous angioscopic studies of the FP artery.,8 All results are expressed as mean \pm SD unless otherwise stated. All calculations were performed using the IBM SPSS Statistics package Version 20 (IBM Corp., Armonk, NY, USA).

\section{Results}

\section{Baseline Characteristics of the Patients}

The patients' characteristics are shown in Table 1. Mean age was $77 \pm 11$ years, and $44 \%$ of the patients were male. In terms of the status of PAD, most patients $(89 \%)$ had critical limb ischemia. More than half $(67 \%)$ of the patients received hemodialysis, and approximately half $(56 \%)$ of the patients had a history of previous coronary artery disease. Table 2 shows the lesion characteristics. Most of the lesions (71\%) were complex, with Trans-Atlantic InterSociety Consensus II classification C or D. Chronic total occlusion was observed in $18 \%$ of lesions. The mean lesion length and mean distal reference vessel diameter were $20 \pm 10 \mathrm{~cm}$ and $4.9 \pm 0.9 \mathrm{~mm}$, respectively. Table 3 shows the procedural characteristics. Mean follow-up duration from stent implantation to angioscopic evaluation was $97 \pm 20$ days. Mean stent diameter and length were $6.7 \pm 0.5 \mathrm{~mm}$ and $10.5 \pm 2.7 \mathrm{~cm}$, respectively. Pre- and postdilatation were performed for all procedures.

\section{Angioscopic Findings}

The details of the angioscopic findings are shown in Table 4. No complications occurred regarding angioscopy. 


\section{A. Dominant Neointimal Coverage Grade}

(\%)100

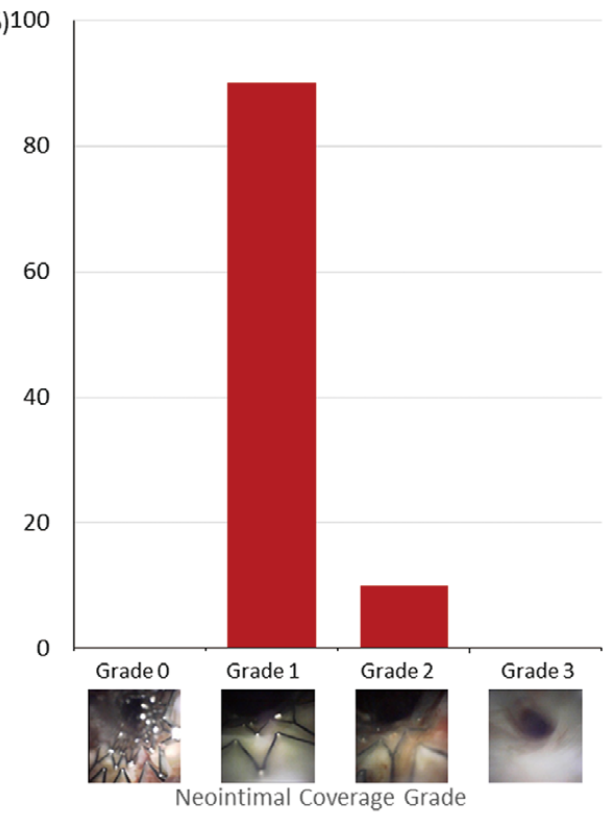

B. Heterogeneity of Neointimal Coverage

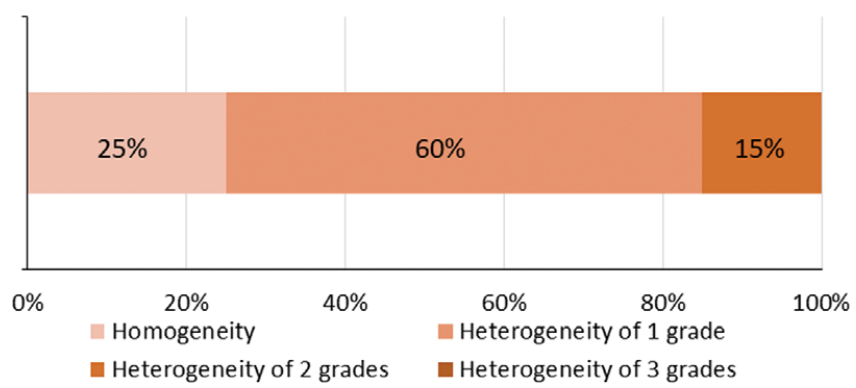

C. Uncover Score

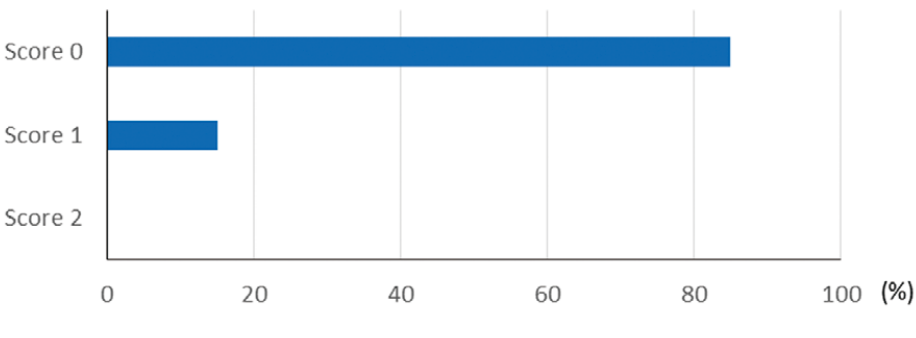

Figure 3. Angioscopic findings 3 months after fluoropolymer-based paclitaxel-eluting stent implantation. (A) Dominant neointimal coverage (NIC): $90 \%$ of stents demonstrated grade 1 dominant NIC, with others showing grade 2 dominant NIC. (B) Heterogeneity of neointimal coverage. Homogeneity, heterogeneity of 1 grade, heterogeneity of 2 grades, and heterogeneity of 3 grades were observed in $25 \%, 60 \%, 15 \%$, and $0 \%$ of the stents, respectively. (C) Extent of uncovered struts: $85 \%$ showed an uncover score 0 , and the remainder had an uncover score 1 . Stents with widely uncovered struts were not detected.

We could not obtain satisfactory intravascular images for 2 stents. Of the 20 stents for which adequate images were available, $18(90 \%)$ showed grade 1 dominant NIC and 2 $(10 \%)$ showed grade 2 dominant NIC (Figure 3A). In terms of the heterogeneity of NIC, homogeneity, heterogeneity of 1 grade, heterogeneity of 2 grades, and heterogeneity of 3 grades were observed in $25 \%, 60 \%, 15 \%$, and $0 \%$ of the stents, respectively (Figure 3B). Regarding the extent of uncovered struts, $85 \%$ of stents showed an uncover score 0 , and the remainder had an uncover score of 1 (Figure 3C). Stents with widely uncovered struts were not detected. Thrombus was observed in all stents. A representative case (case no. 8) is shown in Figure 4.

\section{Discussion}

Our findings revealed that in the FP lesions (1) $90 \%$ of the stents showed grade 1 dominant NIC and $10 \%$ showed grade 2 ; (2) $75 \%$ of the stents demonstrated heterogeneous NIC grade; (3) no stent demonstrated widely uncovered struts; and (4) thrombus adhesion was observed in all stents. To the best of our knowledge, this is the first report describing the early-phase arterial healing of PES in the FP artery evaluated by angioscopy.

In the familial hypercholesterolemic swine model of femoral restenosis, PES exhibited superior inhibition of neointimal proliferation compared with PCS and bare metal stent (BMS) at 90 days after implantation. ${ }^{11}$ In the same study, analysis of neointimal thickness along the stent segments at 90 days by optical coherence tomography demonstrated more uniform distribution in PES compared with PCS and BMS. ${ }^{11}$ In a study comparing the angioscopic findings between PCS and BMS at 2-4 months after implantation, the dominant NIC grade was equally distributed from grade 0 to grade 3 in PCS, and $90 \%$ of BMS showed grade 2 or grade 3 dominant NIC. ${ }^{4}$ Despite the difference in resolution of angioscopy between the current and previous studies, these findings clearly indicate that the distribution of NIC grade of PES differs from that of PCS and BMS. PES might achieve stronger inhibition of neointimal proliferation compared with BMS, and PES did not reveal grade 0 dominant NIC, which was seen in some PCS. Therefore, our angioscopic findings were consistent with the swine model. Because PCS has a polymer-free coating with a paclitaxel dose of $3 \mu \mathrm{g} / \mathrm{mm}^{2}$, paclitaxel elutes to the vessel wall in a relatively short period after implantation. In contrast, PES is coated with a primer polymer poly (n-butyl methacrylate) and an active layer composed of matrix polymer poly (vinylidene fluoride-co-hexafluoropropylene), and these coatings ensure elution of paclitaxel over approximately 12 months at a dose density of $0.167 \mu \mathrm{g}$ paclitaxel $/ \mathrm{mm}^{2}$ stent surface area, which is a lower dose than that eluted from the PCS. ${ }^{3}$ Because PES can elute low-dose paclitaxel steadily for longer, the difference in the effect of paclitaxel over the PES-implanted lesion will be minimized. As a result, PES could prevent healing delayed by excessive cytotoxicity in addition to preventing excessive neointimal proliferation caused by insufficient delivery of paclitaxel to the vessel wall. In this way, thin, uniform and optimal NIC could be obtained. 


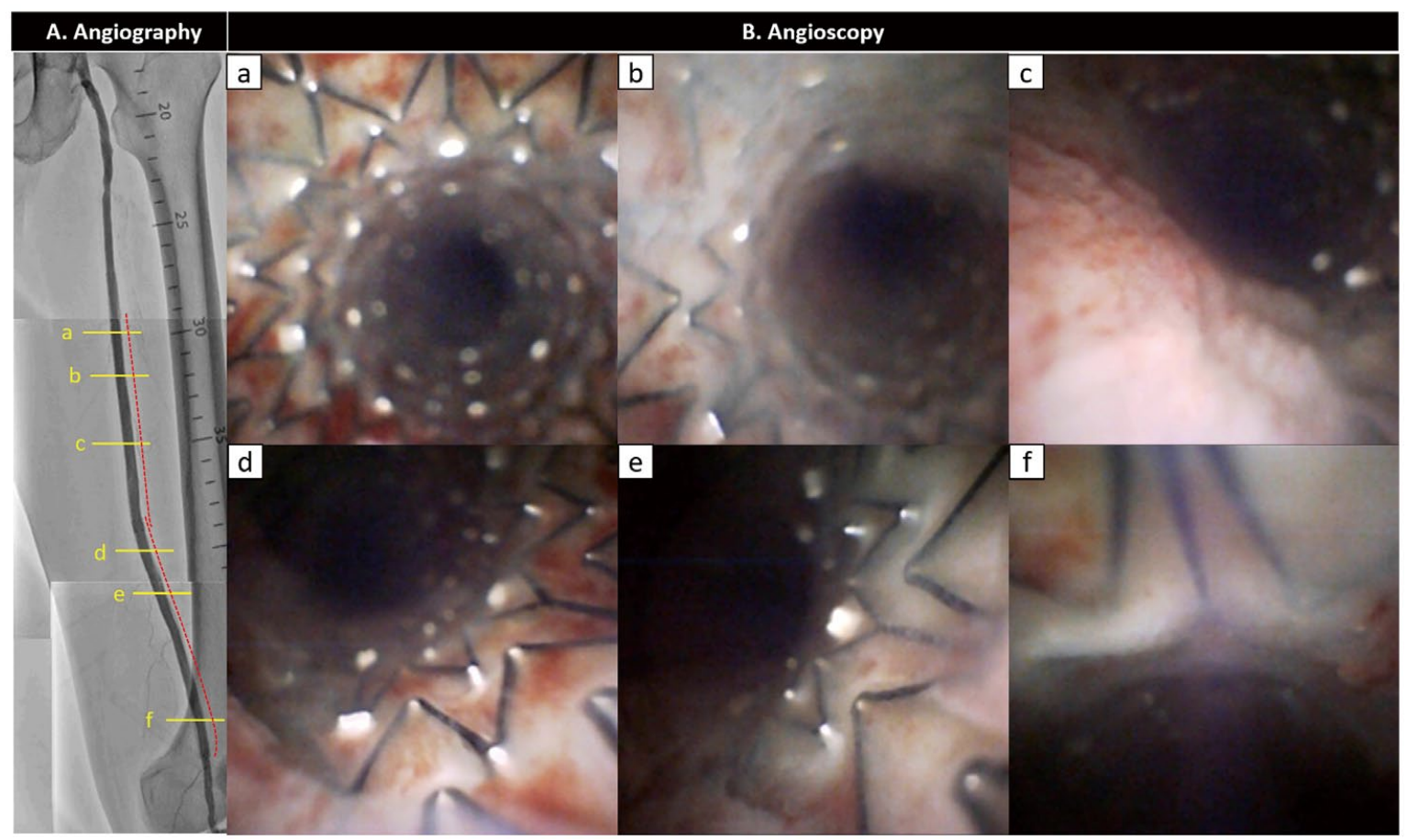

Figure 4. Representative case (no. 8). Angiography and angioscopy were performed 67 days after implantation of a fluoropolymerbased paclitaxel-eluting stent (PES) in the superficial popliteal artery. (A) Angiography shows the stent implantation site is patent. Dashed red line: 2 polymer-coated, PES (proximal: $6.0 \mathrm{~mm} \times 12 \mathrm{~cm}$, distal: $6.0 \mathrm{~mm} \times 12 \mathrm{~cm}$ ). (B) Angioscopic findings at 6 levels (a-f). (a) Grade 1 NIC with red thrombus; (b) grade 1 and grade 2 NIC with red thrombus; (c) grade 3 with red thrombus; (d) grade $1 \mathrm{NIC}$ with red thrombus; (e) grade $1 \mathrm{NIC}$ with red thrombus; (f) grade $2 \mathrm{NIC}$ with red thrombus. NIC, neointimal coverage.

When arterial healing is sufficient and mature re-endothelialization is established, the vessel will exert its own antithrombotic effect via the mature endothelial cells. Therefore, angioscopic thrombus within the stent is thought to be a sign of delayed arterial healing in the chronic phase after stent implantation. ${ }^{9}$ On the other hand, because thrombus is attached to the stented segment at the beginning of arterial healing, thrombus adhesion can be considered an initial phase of arterial healing. ${ }^{12}$ Also, a previous pathological study reported that delayed healing due to excessive fibrin deposition was detected on a PES (Taxus; Boston Scientific) after implantation, and paclitaxel was thought to be the cause of the phenomenon. ${ }^{13}$ In the current study, we observed thrombus adhesion in all PES, which may have been caused by fibrin deposition caused by paclitaxel or which may simply reflect the initial phase of arterial healing. Further investigation beyond 3 months would be necessary to resolve this issue.

One of the most severe adverse events after stent implantation is stent thrombosis (ST), which can result in major amputation. ${ }^{14}$ The IMPERIAL trial demonstrated that the 1-year incidence of ST was $1.7 \%$ in PES and $4.0 \%$ in PCS. ${ }^{3}$ The incidence was numerically lower in PES, although the difference did not reach statistical significance. ${ }^{3}$ Also, Banerjee et al reported that the 1-year incidence of ST was somewhat higher $(10.6 \%)$ in Viabahn stent grafts (Gore Medical, Newark, DE, USA), which is statistically similar to that of PCS $(4.4 \%) .{ }^{15}$ A pathological evaluation demonstrated that neointimal proliferation occurred only at the edges of the stent graft, and NIC never originated within the stent graft. ${ }^{16}$ In terms of the coronary artery, some pathological or optical coherence tomography studies report that widely uncovered struts is one of the mechanisms of ST. ${ }^{10,17-19}$ Based on these findings, non-coverage would increase the risk of ST in FP arteries, making it similar to the risk in coronary arteries. In the current study, widely uncovered struts were not detected in 20 PES at 3 months after implantation in FP lesions. Therefore, we expect that PES will achieve somewhat higher safety outcomes, even though arterial healing may be ongoing.

\section{Study Limitations}

First, it was a single-center, observational study without a control arm. Second, the relationship between angioscopic findings and clinical outcomes has not yet been elucidated in peripheral arteries. Third, angioscopic thrombus does not directly indicate the risk of ST. Fourth, although lesion characteristics and inflammatory status may affect the angioscopic findings at 3-month follow-up, we did not elucidate the relationship between them in the population of this study. Further investigation is necessary to unravel the issue. Finally, in some cases angioscopy does not completely visualize the entire stented segment due to a limitation of the visual field. However, the recently developed angioscope used here has a high resolution of 480,000 pixels, and in this study it allowed us to more clearly observe the intravascular status compared with the older model catheter. 


\section{Conclusions}

Widely uncovered stent struts were not observed by angioscopy at 3 months after PES implantation in FP lesions, even though there was detection of thrombus adhesion.

\section{Acknowledgments}

We thank Mr. Hiroki Oyama, Mr. Kazutoshi Ito and Mr. Yusuke Katagiri for their expertise in performing coronary angioscopic examinations.

\section{Data Availability}

The deidentified participant data will not be shared.

\section{Disclosures}

O.I. received remuneration from Medtronic Japan and Boston Scientific Japan, and T.M. had research funding from Abbott Vascular Japan. The remaining authors have no disclosures to report.

\section{IRB Information}

The Medical Ethics Committee of Kansai Rosai Hospital (reference no. $16 \mathrm{D} 008 \mathrm{~g})$.

\section{References}

1. Iida O, Yokoi H, Soga Y, Inoue N, Suzuki K, Yokoi Y, et al. Cilostazol reduces angiographic restenosis after endovascular therapy for femoropopliteal lesions in the Sufficient Treatment of Peripheral Intervention by Cilostazol study. Circulation 2013; 127: $2307-2315$.

2. Iida O, Takahara M, Soga Y, Nakano M, Yamauchi Y, Zen K, et al. 1-year results of the ZEPHYR registry (Zilver PTX for the Femoral Artery and Proximal Popliteal Artery): Predictors of restenosis. J Am Coll Cardiol Intv 2015; 8: 1105-1112.

3. Gray WA, Keirse K, Soga Y, Benko A, Babaev A, Yokoi Y, et al. A polymer-coated, paclitaxel-eluting stent (Eluvia) versus a polymer-free, paclitaxel-coated stent (Zilver PTX) for endovascular femoropopliteal intervention (IMPERIAL): A randomised, non-inferiority trial. Lancet 2018; 392: $1541-1551$.

4. Ishihara T, Iida O, Awata M, Nanto K, Nanto S, Uematsu M. Angioscopic assessment of early phase arterial repair after paclitaxel-coated nitinol drug-eluting stent implantation in the superficial femoral artery. Circ J 2013; 77: 1838-1843.

5. Ishihara T, Awata M, Sera F, Fujita M, Watanabe T, Iida O, et al. Arterial repair 4 months after zotarolimus-eluting stent implantation observed on angioscopy. Circ J 2013; 77: 1186-1192.

6. den Heijer P, Foley DP, Hillege HL, Lablanche JM, van Dijk RB, Franzen D, et al. The 'Ermenonville' classification of observations at coronary angioscopy-evaluation of intra- and inter-observer agreement: European Working Group on Coronary Angioscopy. Eur Heart J 1994; 15: 815-822.

7. Ishihara T, Tsujimura T, Okuno S, Iida O, Asai M, Masuda M, et al. Early- and middle-phase arterial repair following bioresorbable- and durable-polymer drug-eluting stent implantation: An angioscopic study. Int J Cardiol 2019; 285: 27-31.

8. Ishihara T, Iida O, Awata M, Nanto K, Shiraki T, Okamoto S, et al. Extensive arterial repair one year after paclitaxel-coated nitinol drug-eluting stent vs. bare-metal stent implantation in the superficial femoral artery. Cardiovasc Interv Ther 2015; 30: 51 -56.

9. Mitsutake Y, Ueno T, Yokoyama S, Sasaki K, Sugi Y, Toyama $\mathrm{Y}$, et al. Coronary endothelial dysfunction distal to stent of firstgeneration drug-eluting stents. J Am Coll Cardiol Intv 2012; 5: 966-973.

10. Finn AV, Joner M, Nakazawa G, Kolodgie F, Newell J, John $\mathrm{MC}$, et al. Pathological correlates of late drug-eluting stent thrombosis: Strut coverage as a marker of endothelialization. Circulation 2007; 115: 2435-2441.

11. Gasior P, Cheng Y, Valencia AF, McGregor J, Conditt GB, Kaluza GL, et al. Impact of fluoropolymer-based paclitaxel delivery on neointimal proliferation and vascular healing: A comparative peripheral drug-eluting stent study in the familial hypercholesterolemic swine model of femoral restenosis. Circ Cardiovasc Interv 2017; 10: e004450.

12. Schwartz RS. Pathophysiology of restenosis: Interaction of thrombosis, hyperplasia, and/or remodeling. Am J Cardiol 1998; 81: 14E-17E.

13. Nakazawa G, Finn AV, Vorpahl M, Ladich ER, Kolodgie FD, Virmani R. Coronary responses and differential mechanisms of late stent thrombosis attributed to first-generation sirolimus- and paclitaxel-eluting stents. J Am Coll Cardiol 2011; 57: 390-398.

14. Soga Y, Inoue K, Kuma S. Pathological findings of late stent thrombosis after paclitaxel-eluting stent implantation for superficial femoral artery disease. J Cardiol Cases 2014; 11: 39-41.

15. Banerjee S, Sarode K, Mohammad A, Gigliotti O, Baig MS, Tsai S, et al. Femoropopliteal artery stent thrombosis: Report from the Excellence in Peripheral Artery Disease Registry. Circ Cardiovasc Interv 2016; 9: $\mathrm{e} 002730$.

16. Ishihara T, Iida O, Inoue $\mathrm{K}$, Fujita M, Masuda M, Okamoto S, et al. Histological evaluation of a self-expanding stent-graft 23 months after implantation in the superficial femoral artery. $J$ Endovasc Ther 2017; 24: 746-750.

17. Taniwaki M, Radu MD, Zaugg S, Amabile N, Garcia-Garcia HM, Yamaji K, et al. Mechanisms of very late drug-eluting stent thrombosis assessed by optical coherence tomography. Circulation 2016; 133: $650-666$.

18. Lee SY, Ahn JM, Mintz GS, Hur SH, Choi SY, Kim SW, et al. Characteristics of earlier versus delayed presentation of very late drug-eluting stent thrombosis: An optical coherence tomographic study. J Am Heart Assoc 2017; 6: e005386.

19. Nakamura D, Attizzani GF, Toma C, Sheth T, Wang W, Soud $\mathrm{M}$, et al. Failure mechanisms and neoatherosclerosis patterns in very late drug-eluting and bare-metal stent thrombosis. Circ Cardiovasc Interv 2016; 9: e003785. 